\title{
Design Lower Arm Using Optimum Approach
}

\author{
Adel Mahmoud Bash \\ Department of Mechanical Engineering, College of Engineering, Tikrit University, Salah al_deen, Iraq
}

\begin{abstract}
In the automotive industry, the riding comfort and handling qualities of an automobile are greatly affected by the suspension system. This paper presents the robust design of vehicle suspension arm using stochastic design improvement (SDI) technique based on Monte Carlo simulation. The main goal of this study is to determine the optimum design for the suspension arm. The structural model of the suspension arm was utilizing the Solid works and aluminum alloys (AA7075-T6) are selected as a suspension arm materials. The linear static stress distribution is investigated using the commercial Finite element analysis package, and dynamic analysis was performed using NASTRAN software. SDI has been performed to the design. A target output behavior is selected from the output variables available in the analysis. The result shows that the lower arm design has a higher capability to stand higher pressure as $9.18 \mathrm{MPa}$ with the stress acted on lower arm is $41 \mathrm{MPa}$. The new parameter of material can be chose as optimum result for the lower suspension arm.
\end{abstract}

Keyword: Lower arm, SDI, FEM, Dynamic analysis

\section{Introduction}

Stress analysis activities vary depending on the function and maturity of the phase, an important benefit of performing stress analyses is the ability to determine design sensitivities and to conduct trade studies. (Conle and Mousseau 1991) used vehicle simulation and the finite element results to generate the fatigue life contours for the chassis components using automotive proving ground load history results combined with the computational techniques; They concluded that the combination of the vehicle dynamics modeling, finite-element analysis, and fatigue analysis are the viable techniques for the fatigue design of the automotive components. (Kim et al. 2002) were studied a method for simulating vehicles dynamic loads, but they add durability assessment, For their multibody dynamic analysis they use DADS and a flexible body model. Dynamic stress analysis was performed using MSC. NASTRAN. The fatigue life was then calculated using a local strain approach; The result fatigue life, shows the majority of the fatigue damage occurred over a frequency range that depend on terrain traveled (service or accelerated test course). (Gopalakrishnan and Agrawal 1993) carried out durability analysis of full automotive body structures using an integrated procedure, in which the dynamic simulation software ADAMS was used to generate loading histories, and the Inertia Relief Analysis of MSC.NASTRAN was used to analyze the model and to get displacements and stresses. Designing a robust suspension lower arm is crucial to the success of building the car and requires that suspension components have to be well engineered in aspects of both compactness and crashworthiness, which is defined as a measure of the whole vehicles or its components structural ability to plastically deform and yet maintain a sufficient survival space for its occupants in crashes involving reasonable deceleration loads (Praya and Belwefa 2004). Stochastic design improvement (SDI) is a fast and efficient method for improving the performance of a system. Can be specify the desired target behavior for a system and get multiple alternative solutions that satisfy the target. Most applications of robust design have been concerned with static performance in mechanical engineering and process systems (Zang et al., 2004) whereas the objective of robust design is to optimize the mean and minimize the variability that results from uncertainty represented by noise factors and to test the effect of the variability in different experimental factors using statistical tools. From a technical standpoint the Statistical process control (SPC) and Statistical experimental design (SED) are two methods has been used as a robust design of an automotive suspension arm to improve quality and productivity (Rahman, 1994). The inputs into such programs must include a complete description of the forces acting on the components via dynamic modeling (Frimpong et al., 2005). In this paper, finite element techniques have been used as a tool to model the mechanical properties of the suspension arm. Three-dimensional linear tetrahedron solid elements 10 nodes tetrahedral (TET10) used for the initial analysis based on the loading conditions. Convergence of stress energy was considered as the criteria to select the mesh size and predict the dynamic behavior of suspension arm; SDI has been performed to the design using robust design codes. A target output behavior is selected from the output variables available in the FEM. In the optimization process for the design all the parameters have been set as hard target for stress and design variable for material. 


\section{Optimization Technique}

Optimization problems in practice depend mostly on several model parameters, noise factors, uncontrollable parameters, etc., which are not given fixed quantities at the planning stage. Due to several types of stochastic uncertainties (physical uncertainty, economic uncertainty, statistical uncertainty, and model uncertainty) these parameters are modeled by random variables having a certain probability distribution. In most cases at least certain moments of this distribution are known. Robust Design provides the means to quickly sort through this information and indicate the variables that have the most significant correlations, and therefore most impact the product's performance. Correlation is a concept different from of sensitivity in that collective changes in variable values are considered. Correlation between two variables expresses the strength of the relationship between these variables by taking into account the scatter in all the other variables in a system. It is possible to compute correlations between any pair of variables (input-output, output-output, etc.). Knowledge of the correlations in a system is equivalent to the understanding of how that system works. The above correlations are used in Robust Design for results interpretation and are labeled as linear and non-linear correlations on output plots. Pearson's correlation coefficient measures the linear correlation between variables. For two stochastic variables, $\mathrm{x}$ and $\mathrm{y}$, their Pearson, or linear correlation is expressed as follows

$$
\mathrm{r}=\frac{\sum_{\mathrm{i}}\left(\mathrm{x}_{\mathrm{i}}-\mathrm{u}_{\mathrm{x}}\right)\left(\mathrm{y}_{\mathrm{i}}-\mathrm{u}_{\mathrm{y}}\right)}{\sqrt{\sum_{\mathrm{i}}\left(\mathrm{x}_{\mathrm{i}}-\mathrm{u}_{\mathrm{x}}\right)^{2}} \sqrt{\sum_{\mathrm{i}}\left(\mathrm{y}_{\mathrm{i}}-\mathrm{u}_{\mathrm{y}}\right)^{2}}}
$$

Where $u$ is the mean value, the values of the Pearson correlation range from -1 to 1 . A value close to either 1 or -1 indicates a strong linear correlation. Values close to zero indicate the variables are uncorrelated.

The Spearman rank correlation coefficient rs, is then computed as the linear correlation coefficient between the ranks $\mathrm{Ri}$ of the xis and the ranks $\mathrm{Si}$ of the yis, the rs is expressed as follows

$$
\mathrm{r}_{\mathrm{S}}=\frac{\sum_{\mathrm{i}}\left(\mathrm{R}_{\mathrm{i}}-\mathrm{u}_{\mathrm{r}}\right)\left(\mathrm{S}_{\mathrm{i}}-\mathrm{u}_{\mathrm{S}}\right)}{\sqrt{\sum_{\mathrm{i}}\left(\mathrm{R}_{\mathrm{i}}-\mathrm{u}_{\mathrm{R}}\right)^{2}} \sqrt{\sum_{\mathrm{i}}\left(\mathrm{S}_{\mathrm{i}}-\mathrm{u}_{\mathrm{S}}\right)^{2}}}
$$

For the design of the lower arm suspension, the application of Stochastic Design Improvement (SDI) will be use which is in the MSC.Robust Design software. SDI is a fast and efficient capability to improve a system design so that its most probable behavior coincides with specified target values.

This process continues until either the target value or a physical limit for the design variable is reached. This is normally accomplished with 4 to 6 sets of 15 analysis runs. The key feature of SDI is that it operates on a full FE model, which incorporates tolerances, and not on a simplified surrogate. SDI surpasses classical optimization techniques in terms of performance and computational cost. Figure 1 shows the flows of steps that will be done in the SDI for the lower arm suspension design.

\section{Motion for Suspension System of Automobile}

Natural frequency is the rate of energy interchange between the kinetic and the potential energies of a system during its cycle motion. As the mass pass through the static equilibrium position, the potential energy is zero (Dimarogonas, 1996). The natural frequency is expresses as Eq. (3).

$$
w_{n}=\sqrt{\frac{k}{m}}
$$

Where: $\quad w$ is natural frequency

$k$ is coefficient of spring

$m$ is mass

The chassis natural frequency is used the suspension rate and chassis mass and expressed as in Eq. (4),

$$
w_{n}=\sqrt{\frac{k_{s}}{m_{c}}}
$$

where

$$
\begin{gathered}
w_{n} \text { is natural frequency for the car } \\
k_{s} \text { is coefficient of spring } \\
\mathrm{m}_{\mathrm{c}} \text { is mass of the car }
\end{gathered}
$$

For the wheel natural frequency $\omega \mathrm{w}$, it's necessary to take into account Ks and Kt because the wheel oscillates the suspension and tire springs. Although these two springs are on opposite side of the wheel $/ \mathrm{hub} / \mathrm{knuckle}$ mass, the mass would feel the same force if the two springs were in parallel on one side of the mass. In other words, the two springs, $\mathrm{Ks}$ and $\mathrm{Kt}$, are in parallel and their composite rate is their sum. 


$$
w_{w}=\sqrt{\frac{k_{s}+k_{t}}{m_{w}}}
$$

Where $\quad w_{w}$ is the natural frequency of the wheel

$$
\begin{aligned}
& k_{s} \quad \text { is the coefficient of spring } \\
& k_{t} \quad \text { is coefficient of tire } \\
& m_{w} \text { is mass of the wheel }
\end{aligned}
$$

\subsection{Model Description}

Vehicle suspension is a mechanism locating between the sprung mass (vehicle body) and the unsprung masses (wheels) of the vehicle. The suspension provides forces between these two masses of the vehicle according to certain state variables of the vehicle. A good car suspension system should have satisfactory road holding ability, while still providing comfort when riding over bumps and holes in the road. When the bus is experiencing any road disturbance the bus body should not have large oscillations, and the oscillations should dissipate quickly. A simple three-dimensional model of suspension arm was modeling by used Solid Works software as shown in Figure 2.

\subsection{Mechanical Properties}

Material model and material properties play an important role in the result of FE method. The material properties are one of the major inputs, which is definition of how the material behaves under the cyclic loading conditions. The materials parameters required depend on the analysis methodology being used. The mechanical properties of 7075-T6 aluminum alloy are shown in Table 1.

\section{Results and Discussion}

\subsection{Modeling and Simulation}

The lower arm suspension is one of the important parts in the suspension system. A specific area of constraint has been set into the design in order to get a precise result. TET10 has been used in the finite element modeling using MSC. PATRAN. These analyses were preformed iteratively at different mesh global length until the appropriate accuracy obtained. The convergence of the stresses was studied as the mesh global length was refined in the analysis. The mesh global length of $0.1 \mathrm{~mm}$ was chosen and the pressure of $8 \mathrm{MPa}$ was applied at the end of the bushing that connected to the tire. The other two bushing that connected to the body of the car are constraint. The pressure that has been applied is based on (Al-Asady et al. 2008). The three-dimensional FE model, loading and constraints of suspension arm is shown in Figure 3.

\subsection{Effects of the Mesh Types}

The stress histories calculated using the linear static analysis method are usually the most accurate and are commonly used by members of the finite element community as a reference to evaluate the accuracy of the stochastic design improvement. The linear static stress analysis was performed utilizing MSC.NASTRAN to determine the stresses result from finite element model. The material models utilized of elastic and isotropic material. The tetrahedral element TET10 was use for the mesh analysis Figure 4. The convergence of the finite element model of the structure was tested for TET10 and 5 different mesh sizes. Figure 5 shows the von Mises stress contour for TET10 element. The linear elastic analysis results including maximum principal stress, von Mises stress, and Tresca stress are tabulated in Table 2. The convergence of the stress was considered as the main criteria to select the mesh type. The finite element mesh was generated using TET10 for various mesh global length. From the stress analysis, the result shows that the white area of the design is the lowest predicted stress acted on the lower arm suspension design. Therefore, the area can be made as a guide in the future process of modifying or optimizing the design. It is also important to make sure that the critical points on the design which have the highest predicted stress should be look carefully in the process of modifying and optimizing the design in order to avoid any failure in the future usage of the lower arm design.

Figure 6 shows the predicted results of stresses at the critical location of the suspension arm. It can be seen that the smaller the mesh size capture the higher predicted stresses. It is also observed that mesh size of $0.1 \mathrm{~mm}$ (54178 elements) has obtained the maximum stresses, which is almost flatter in nature. The maximum stress obtained of 50.3, 52.2 and 56.3 MPa for von Mises stress, Tresca and Maximum principal stress method respectively. The maximum principal stress method occurred highest stresses through the global length range. Thus TET10 and maximum principal stress method are selected for linear static and dynamic analyses of the 
suspension arm. Thus TET10 at mesh size 0.1 and maximum principal stress method are selected for linear static and stochastic design improvement of the suspension arm.

\subsection{Dynamic Analysis of Lower Arm}

Dynamic analysis is focused on the eigen-frequencies and mode shapes. From a physical point of view an initial excitation of an undamped system causes to vibrate and the system response is a combination of eigenmodes, where each eigenmode oscillates at its associated eigen-frequency (Patrik, 2001). Modal analysis is usually used to determine the natural frequencies and mode shapes of a component. It can be used as the starting point for dynamic analysis. The finite element analysis codes usually used several mode extraction methods. The Lanczos mode extraction method is used in this study. Lanczos is the recommended method for the medium to large models. In addition to its reliability and efficiency, the Lanczos method supports sparse matrix methods that significantly increase computational speed and reduce the storage space. This method also computes precisely the eigenvalues and eigenvectors. The number of modes was extracted and used to obtain the suspension arm stress histories, which is the most important factor in this analysis. Using this method to obtain the first 10 modes of the suspension arm, which are presented in Table 3 and the shape of the mode are shown in Figure 7. It can be seen that the working frequency $(80 \mathrm{~Hz})$ is far away from the natural frequency $(233.26 \mathrm{~Hz})$ of the first mode. A sample of the resulting eigenvalue/eigenvector from the suspension arm is shown in Table 4.

\subsection{Stochastic Design Improvement}

A stochastic simulation generates multiple scenarios of a model by repeatedly sampling values from the probability distributions for the uncertain variables. The stochastic simulation takes the uncertainty of variables into account to determine the level of uncertainty in the outputs. The more stochastic variables a model contains, the more realistic it is. From the Figure 8, the relative influence of tolerances in input variables on the scatter in a particular functionality (output) can be obtained. The pressure gives the largest influence on the stress value followed by the Poisson ratio, modulus of elasticity, and density respectively. This result is very logical and showed that the design is functioning correctly. Figure 9 shows ant hill scatter for stress against materials. It can be seen that there is less interaction (correlation) between them. Linear and non-linear correlation betwee them are obtained negative (linear cor. $=-0.594$ and non-linear cor. $=-0.583$ ). Figure 10-11 show the Ant hill correlation between the maximum principal stress and von Mises stress versus pressure respectively. It can be seen that the correlation between the stress and load scale factor are strongly correlation between them (linear cor. $=0.994$ and non-linear $=0.988$ for maximum principal stress and linear cor. $=1.0$ and non-linear $=1.0$ for von Mises stress. It is to be more dominant that's confirming the result in the pie chart Figure 8.

In the optimization process for the design all the bounds of function study have been set as follows.

1) Max SDI step is 5 .

2) Design variable: Modulus of Elasticity, Poisson Ratio, Density, Pressure.

3) Hard target: Von Mises Stress (50.3MPa).

The results of SDI shows that there are multiple samples from the ant hill scatter plot that give the value of the parameter to use in the optimization process. So the outcome from the SDI had been selected and it can be shown in Table 5. Figure 12 show that lower arm design has a higher capability to stand higher pressure as 9.18 $\mathrm{MPa}$ with the stress acted on lower arm is $41 \mathrm{MPa}$.

\section{Conclusion}

An example was attempted on how robust design technique could be applied in the design stage of the product optimum process so as to maximize product reliability. A detailed model of suspension arm has been developed using finite element techniques. The tetrahedral elements (TET10) is used for the initial analysis then used for the solid mesh. Sensitivity analysis was performed to determine the optimum element size. It can be seen that the TET10 at mesh size 0.1 capture highest moment levels von Mises stress for this reason used to dynamic analysis. The results of the frequency are shown 10 modes and several deformation shapes and from the results proved that the control suspension arm model has been predicted the dynamic behavior. A robust design of lower arm suspension using stochastic optimization is presented. From the analysis, several conclusions can be drawn as follows; The design capability to endure more pressure with lower predicted stress is identified through the optimization process, A lower density and modulus of elasticity of material can be reconsidered in order to optimize the design, and The area of the design that can be altered for the optimization and modification is identified through the stress analysis result. 


\section{References}

Al-Asady, N.A., Abdullah, S., Arrifin, A.K., Rahmman, M.M. and Beden, S.M. (2008). Improving the automotive lower suspension arm durability using finite element analysis, Seminar on Engineering Mathematics. Universiti Malaysia Pahang of Department of Mechanical and Materials Engineering.

Conle, F.A and Mousseau C.W. (1991). Using vehicle dynamic simulation and finite element result to generate fatigue life contours for chassis component. International Journal of Fatigue, 13(3): 195 - 205.

Dimarogonas, A. (1996). Vibration for Engineers. Second Ed. New York: Prentice Hall.

Frimpong, S., Yafei, H. and Awuah-Offei, K. (2005). Mechanics of cable shovel-formation interactions in surface mining excavations. Journal of Terramechanics 42: 15-33.

Gopalakrishnan, R., and H. N. Agrawal. (1993). Durability Analysis of Full Automotive Body Structures. SAE International Congress and Exposition, Detroit, MI, March.

Kim, H.S, Yim, H.J and Kim, C.M. (2002). Computational durability prediction of body structure in prototype vehicles. International Journal of Automotive Technology, 3(4): 129-136.

Patrik, H. (2001). FE-adaptivity and a nonlinear eigenproblem algorithm. Department of Structural Mechanics, Chalmers University of Technology, Sweden.

Praya, Prasad and jamel E. Belwefa. (2004). Vehicle crashworthiness and occupant protection, American Iron and steel institute 2000 Town center Southfield, Michigan 48075.

Rahman P. (1994). Robust Design of an Automotive Suspension System, M.S. Thesis. Michigan Technological University, USA, 1994.

Zang C., M.I. Friswell, and J.E. Mottershead. (2004). A review of robust optimal design and its application in dynamics, Computers \& Structures, vol.83, no.4-5, pp. 315-326, 2004.

Table 1. Mechanical properties of aluminum alloy 7079-T6

\begin{tabular}{ccccc}
\hline Material & $\begin{array}{c}\text { Young's Modulus } \\
\text { (GPa) }\end{array}$ & $\begin{array}{c}\text { Poisson's } \\
\text { ratio }\end{array}$ & $\begin{array}{c}\text { Tensile strength } \\
\text { (MPa) }\end{array}$ & $\begin{array}{c}\text { Yield strength } \\
\text { (MPa) }\end{array}$ \\
\hline $\begin{array}{c}\text { Aluminum alloy } \\
\text { AA7079-T6 }\end{array}$ & 72 & 0.33 & 503 & 572 \\
\hline
\end{tabular}

Table 2. Variation of stresses concentration at the critical location of the suspension arm for TET10 mesh

\begin{tabular}{cccccc}
\hline $\begin{array}{c}\text { Mesh size } \\
(\mathbf{m m})\end{array}$ & $\begin{array}{c}\text { Total } \\
\text { nodes }\end{array}$ & $\begin{array}{c}\text { Total } \\
\text { Elements }\end{array}$ & $\begin{array}{c}\text { Von Mises } \\
(\mathbf{M P a})\end{array}$ & $\begin{array}{c}\text { Tresca } \\
\mathbf{( M P a )}\end{array}$ & $\begin{array}{c}\text { Max Principal } \\
\text { Stress (MPa) }\end{array}$ \\
\hline 0.1 & 96080 & 54178 & 50.3 & 52.2 & 56.3 \\
0.3 & 10041 & 4676 & 50.2 & 51.3 & 54.2 \\
0.6 & 5889 & 2665 & 48.9 & 50.6 & 52.2 \\
1.0 & 5436 & 2465 & 47.4 & 48.2 & 50.7 \\
1.5 & 3186 & 1409 & 45.3 & 35.7 & 49.9 \\
\hline
\end{tabular}


Table 3. Natural frequency of lower arm

\begin{tabular}{cc}
\hline No. of Mode & $\begin{array}{c}\text { Natural Frequency } \\
(\mathbf{H z})\end{array}$ \\
\hline 1 & 233.26 \\
2 & 424.88 \\
3 & 889.65 \\
4 & 1114.7 \\
5 & 1388.4 \\
6 & 1546.4 \\
7 & 1660.6 \\
8 & 2380.7 \\
9 & 2498.3 \\
10 & 2535.2 \\
\hline
\end{tabular}

Table 4. Maximum displacements from modal analysis

\begin{tabular}{clll}
\hline Mode No & T1 $(\boldsymbol{\mu m})$ & $\mathbf{T 2}(\boldsymbol{\mu m})$ & $\mathbf{T 3}(\boldsymbol{\mu m})$ \\
\hline 1 & 146.89 & 5825.3 & 10135.5 \\
2 & 629.77 & 6349.31 & 1088.46 \\
3 & 1234.45 & 5807.32 & 950.36 \\
4 & 1568.87 & 7615.65 & 1572.16 \\
5 & 2854.58 & 1533.89 & 490.42 \\
6 & 1377.12 & 6320.35 & 1781.14 \\
7 & 1262.52 & 5506.094 & 1614.21 \\
8 & 2178.52 & 5343.608 & 2623.39 \\
9 & 1925.07 & 5991.516 & 2042.43 \\
10 & 2586.68 & 4238.355 & 2572.20 \\
\hline
\end{tabular}

Table 5. Comparison of the design parameter before and after optimization

\begin{tabular}{lcc}
\hline Design parameter & FEM & SDI \\
\hline Modulus of elasticity & $71.7 \mathrm{GPa}$ & $69.1 \mathrm{GPa}$ \\
Density & $2.7 \mathrm{~g} / \mathrm{cc}$ & $2.489 \mathrm{~g} / \mathrm{cc}$ \\
Poisson Ratio & 0.33 & 0.3428 \\
Pressure acted on the design & $8 \mathrm{MPa}$ & $9.18 \mathrm{MPa}$ \\
Maximum von Mises stress & $50.3 \mathrm{MPa}$ & $41.0 \mathrm{MPa}$ \\
\hline
\end{tabular}




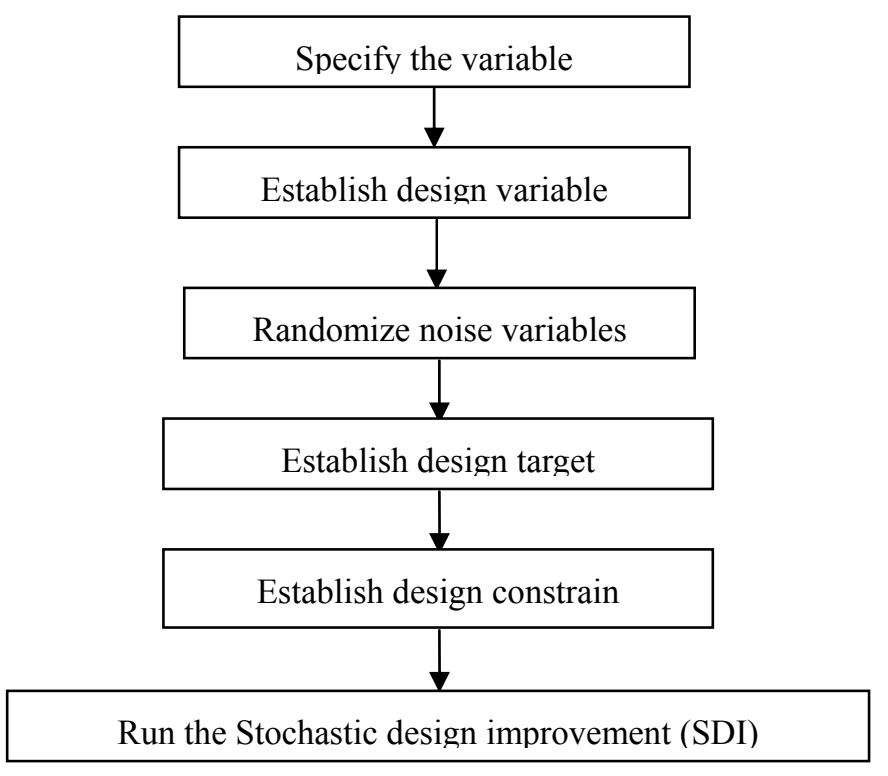

Figure 1. Flow chart of steps in SDI

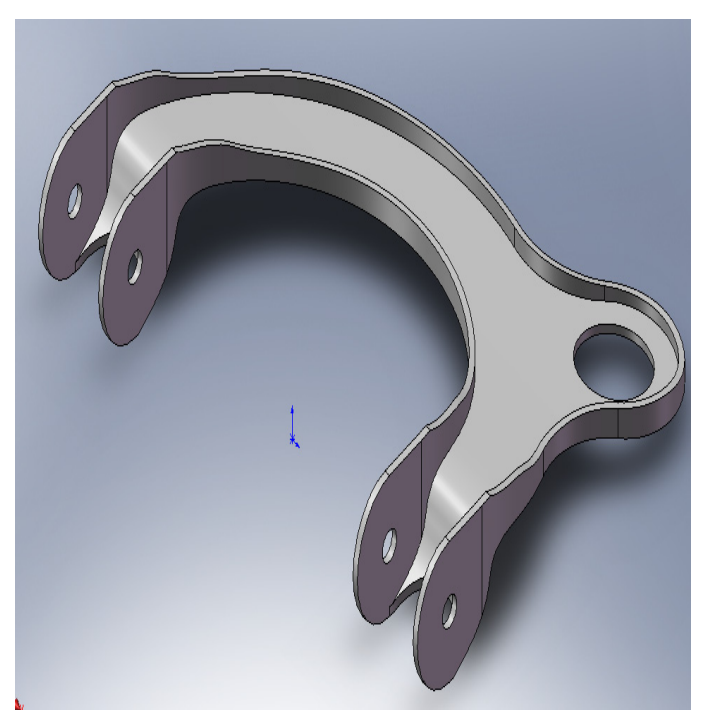

(a) Structural model

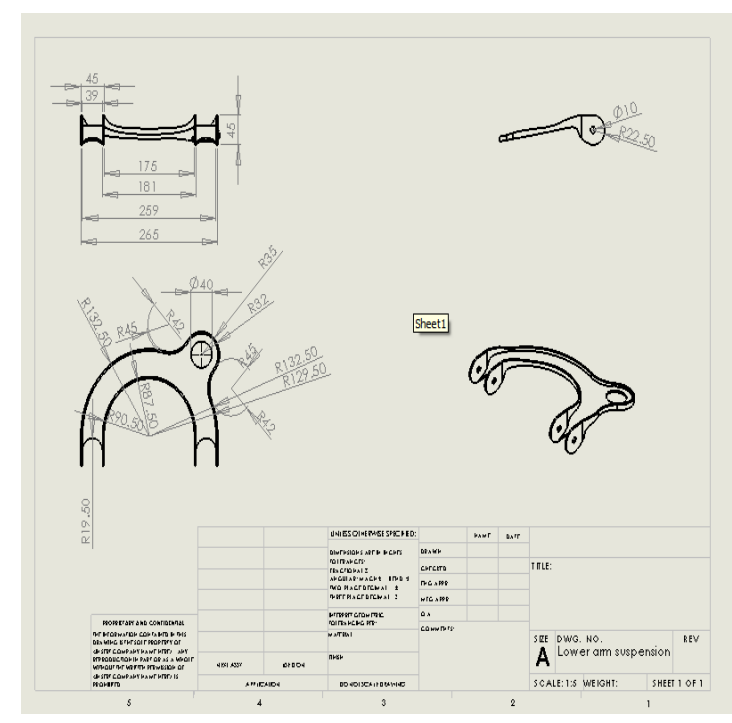

(b) Overall dimension

Figure 2. Structural mode 




Figure 3. Three-dimensional FE model, loading and constraints

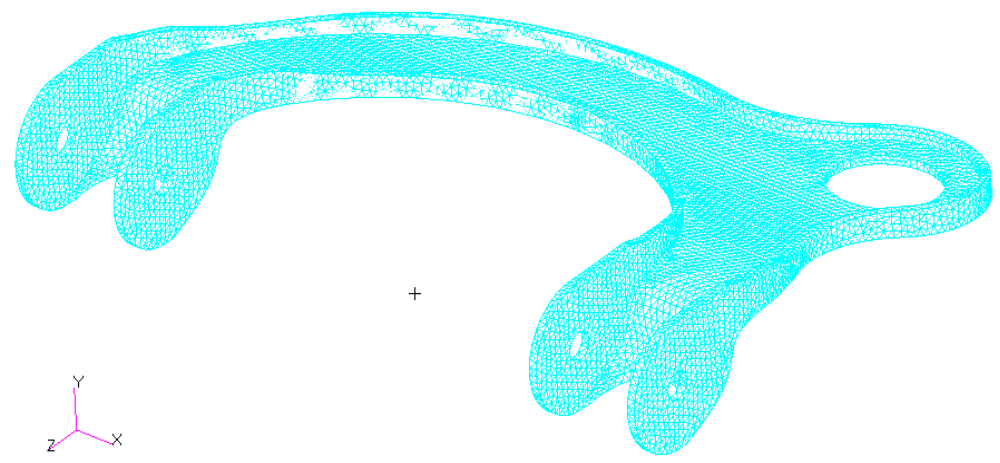

Figure 4. TET10, 54178 elements and 96080 nodes

Figure 5. von Mises stresses contour for TET10 


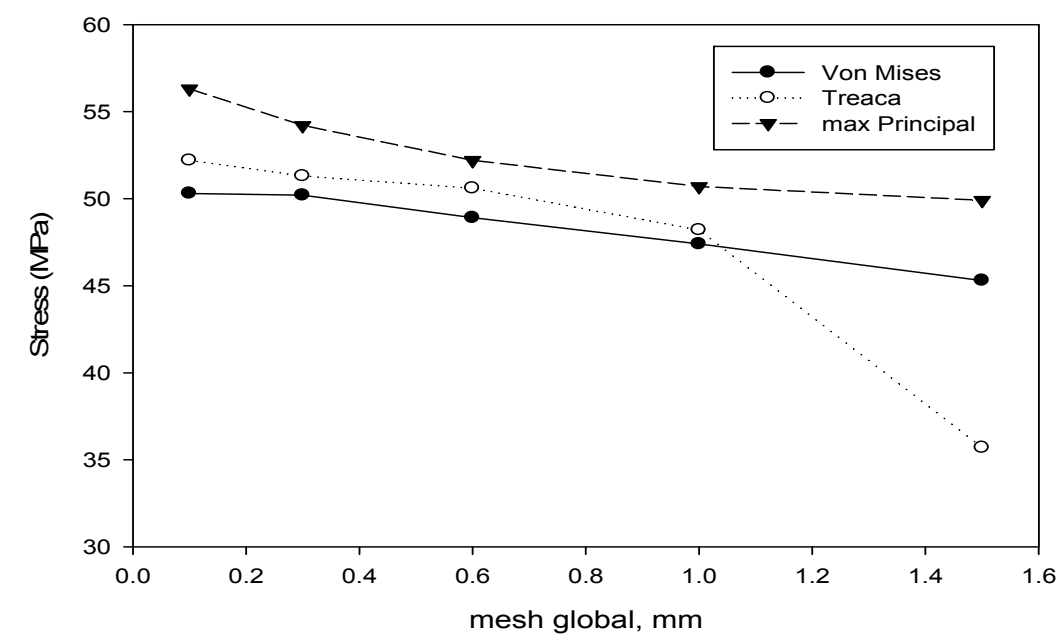

Figure 6. stress contour for TET10
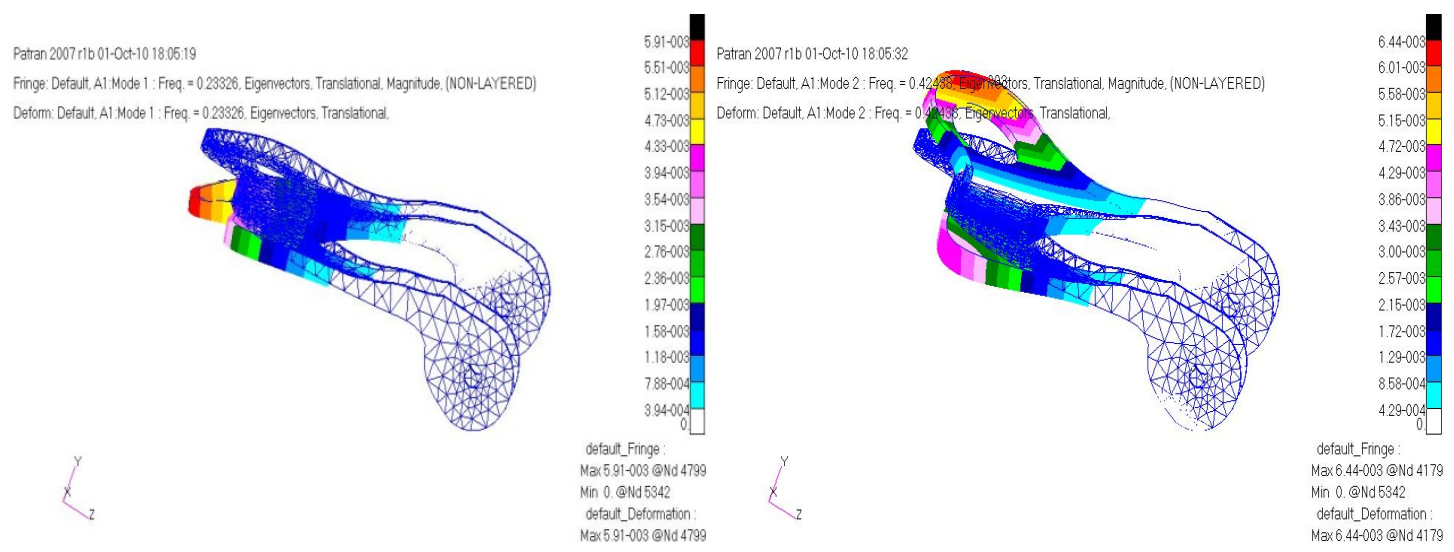

Pattan 2007 r16 01-0ct-10 18:05:43

Fringe: Defaut.,A1:Mode 3 : Freq. = 0.88965 . Eigenvectors, Translational, Magnitude, (NON-LAYERED] Deform: Default. A1: Mode 3: Freq. = 0.88965, Eigenvectors. Translational,
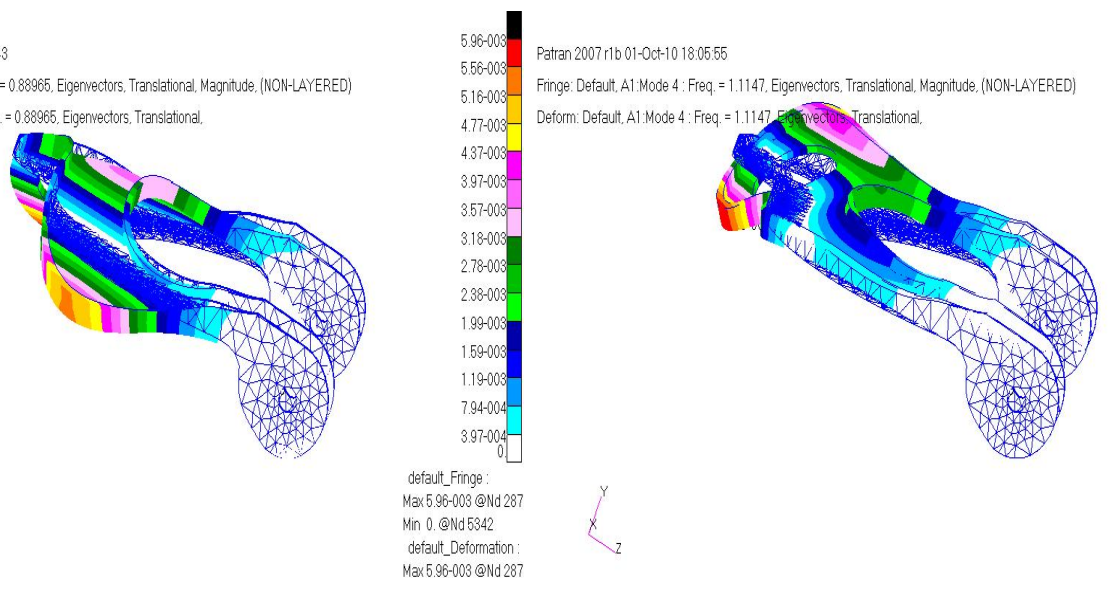

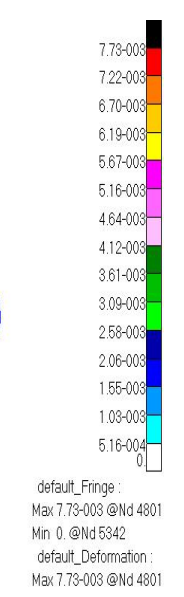


Patan 2007 r t $001-000$-10 18:06:13

Fringe: Default. A1:Mode 6: Freq. = 1.5464, Eigenvectors, Translationa, Nagritude, (NON-LAYERED)

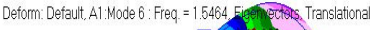

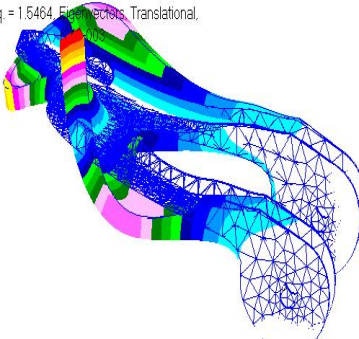

$y_{2}$

Pattan 2007 110 01-0ct-10 18:06:21

Fringe: Defaut A1: Mode 7: Freq = 1.6606 Einegrvectors. Translational, Magnitude, (NON-LAYERED)

Deform: Defaut: A1: Mode 7 : Freq
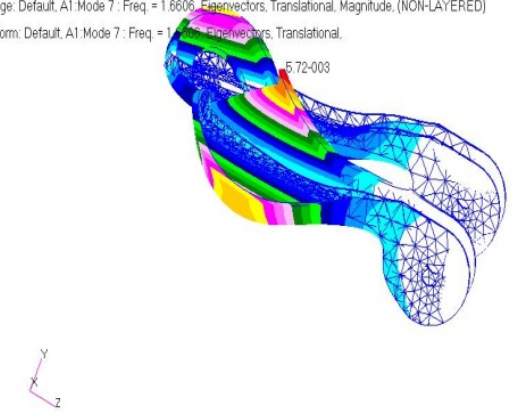
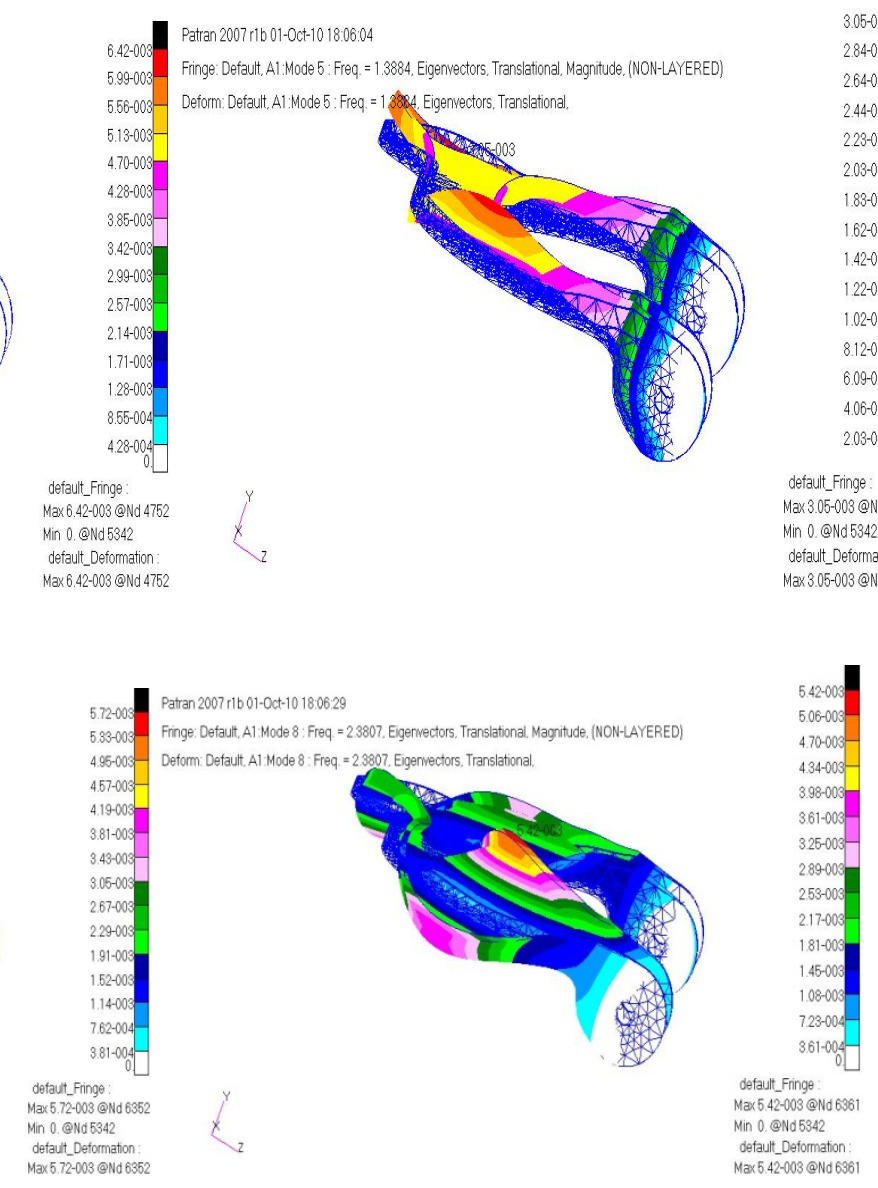

Vin 0.@Nd5342

Max5.42-003 @Nd 698
Patran 2007 r1b 01-0ct-10 18:06:40

Fringe: Defaut, A1: Node 9: Freq, = 2.4983, Eigenvectors., Trensletional, Magnitude, (NON-LAYERED)

Deform Default A1 Wode 9 Freq $=24983$ B 10 - 003

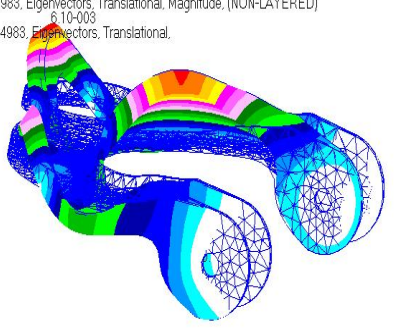

6.100003 - Fatran 2007 r1 01-0ct-10 18:07:29

Fring: Defaut. A1: Mode 10: Freq. = 2.5352, Eigenvectors, Trans ational, Magnitude, (NON-LAYERED) Deform: Defaut.,A1: Mode 10: Freq. = 2.535.5

$4.47-003$
$4.07-003$
$3.66-003$
$325-003$
$2.85-003$
2.44003
$2.03-003$
$1.63-003$
$1.22-009$
8.13004
$4.07-004$

default_Fringe:

Max6.10-003@Nd 278

Min 0. @QNd 5342

default_Leformation:

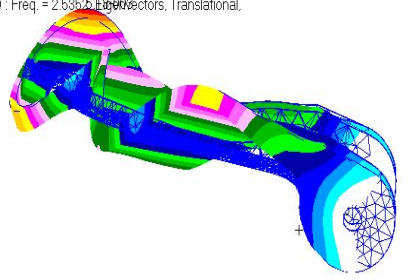

Max3.05-003@Nd28

defaut_Cefomation

Figure 7. frequency mode shape of lower arm 
Subcase 1 Stress Max Principal A (Output 2)

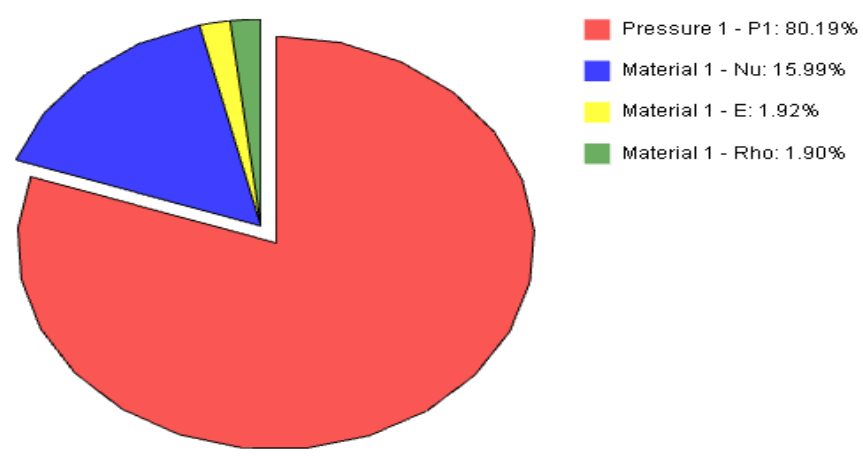

Figure 8. Pie chart of factors that influence to the stress value in the design

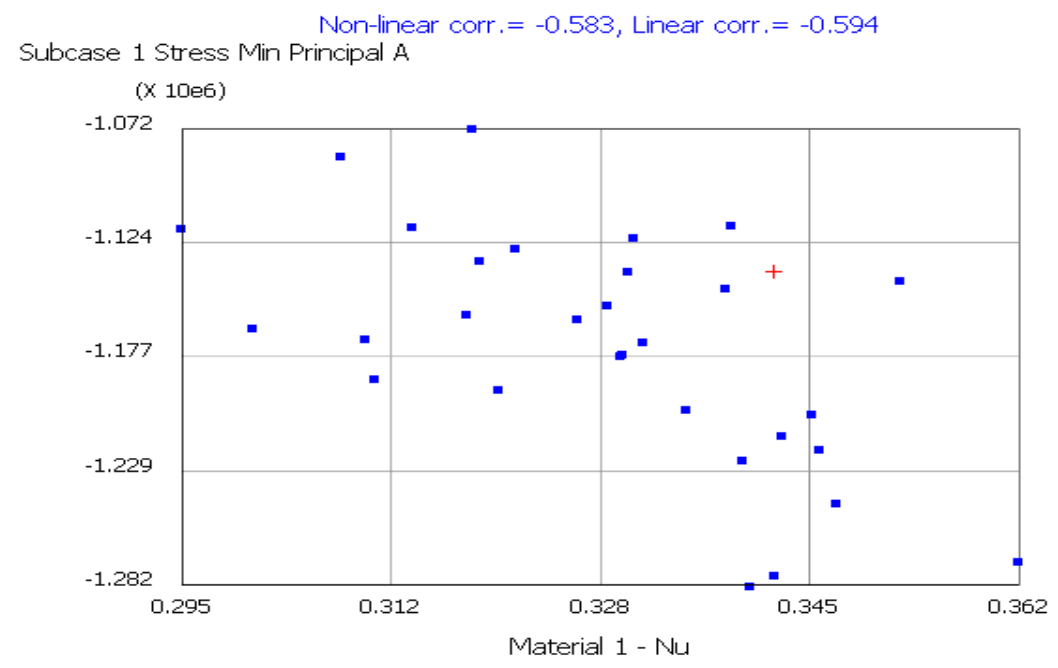

Figure 9. Ant hill scatter plot for stress VS Poisson ratio

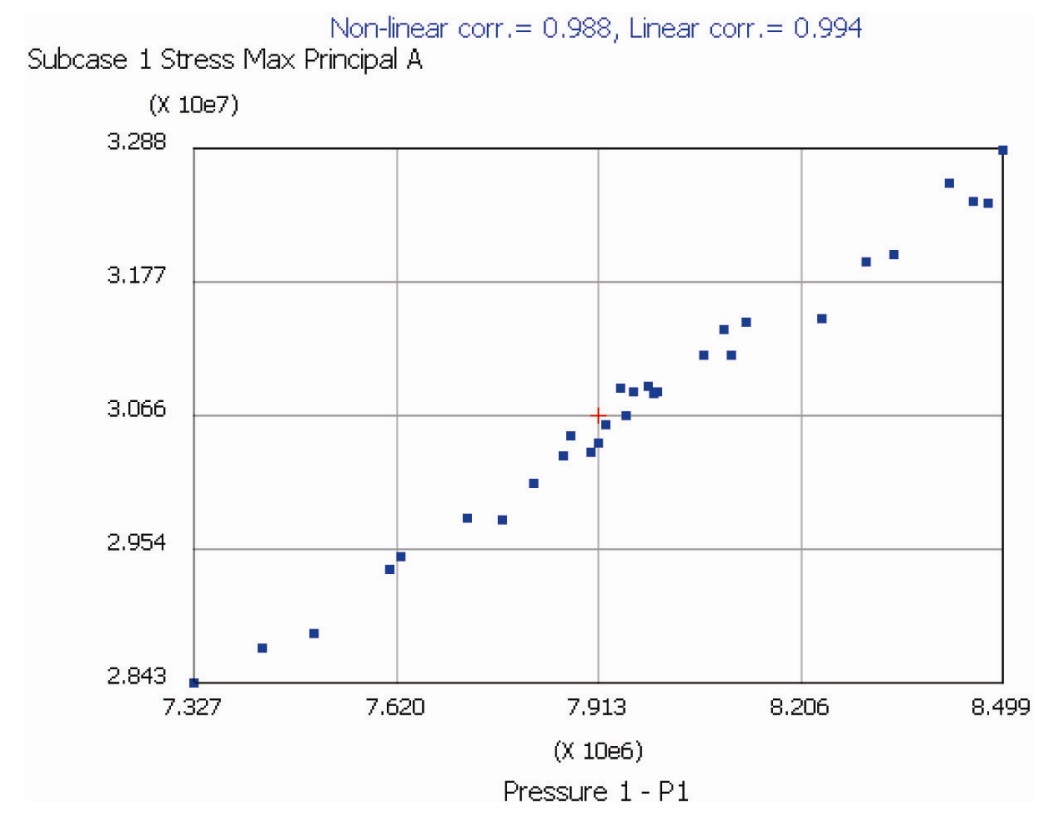

Figure 10. Ant hill scatter plot for stress VS pressure 
Non-linear corr $=1.000$, Linear corr $=1.000$

Subcase 1 Stress Max Von Mises

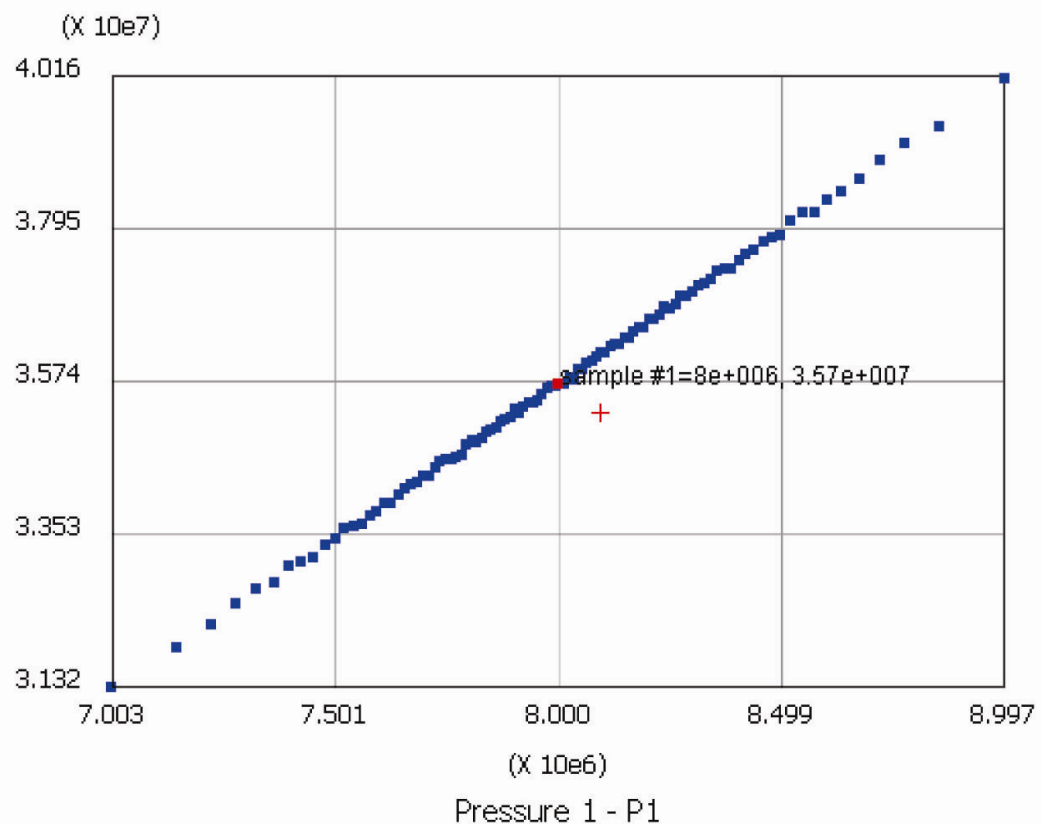

Figure 11. Ant hill scatter plot for stress VS pressure Non-linear corr $=0.999$, Linear corr $=1.000$

Subcase 1 Stress Max Von Mises

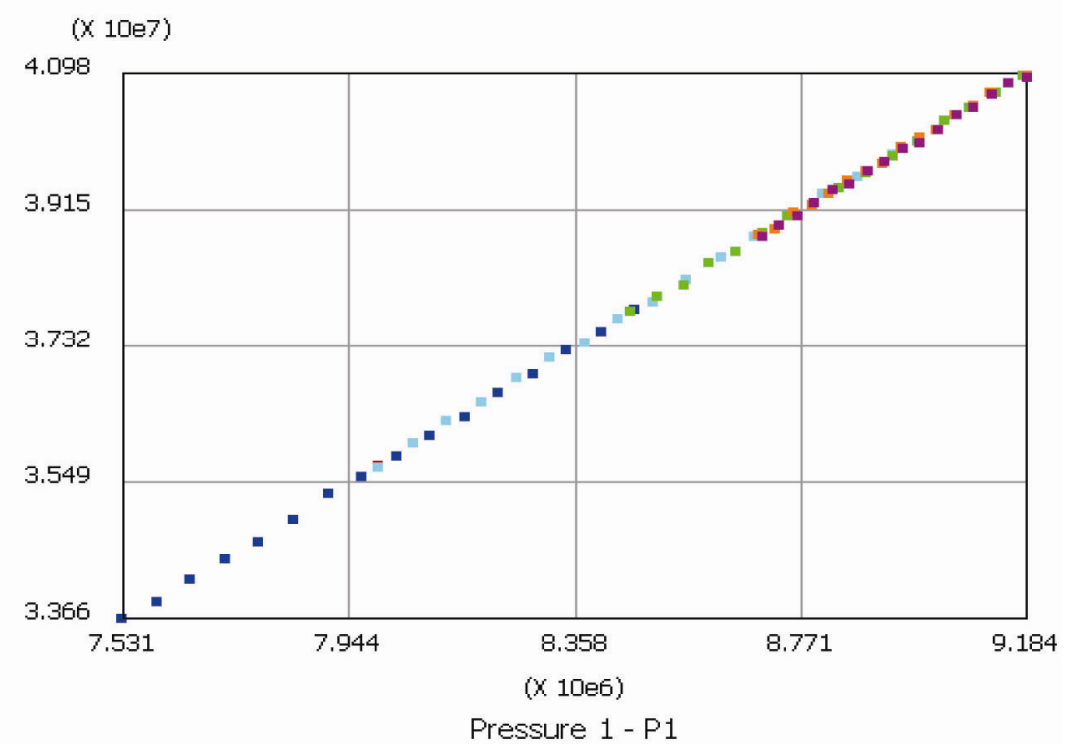

Figure 12. Ant hill scatter plot for stress VS pressure (After SDI) 